\title{
Microbial Community of Human Skin and its Role Vitiligo Disease
}

\author{
Mostafa AA Moustafa ${ }^{1}$ and Samira R Mansour ${ }^{2 *}$ \\ ${ }^{1}$ Faculty of medicine, 6 October University, Egypt \\ ${ }^{2}$ Botany Department, Suez Canal University, Egypt
}

*Corresponding author: Samira R Mansour, Professor of Microbiology, Botany Department, Faculty of Science, Suez Canal University, Ismailia, Egypt,

To Cite This Article: Mostafa AA Moustafa, Samira R Mansour, Microbial Community of Human Skin and its Role Vitiligo Disease. Am J Biomed Sci \& Res. 2021 - 12(1). AJBSR.MS.ID.001705. DOI: 10.34297/AJBSR.2021.12.001705.

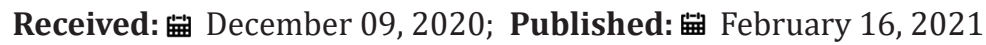

\begin{abstract}
Skin harbor a diverse group of microorganisms that live all together in a harmony forming a biofilm of human skin and named "skin microbiome". This microbial community is dynamic in determining the health of our skin. Skin microbiome is involved in different function in which protection for our body from harmful diseases considered the most important function. Skin's healthiness can be determined by the presence of specific dominating types of microbiome. Skin microbiome can be categories into three groups of microbiome: 1. commensal (harmless and very rarely associated with disease), and 2. symbiotic (beneficial co-existence with the host without any harm), 3. opportunistic pathogens (harm and induce skin disease). Disturbance in skin microbiome is related to gut microbiome dysbiosis which leads to many skin diseases including vitiligo. This disease is resulted from destruction of melanocytes which destroy its capability for melanin production and white skin patches will appear. Theory for vitiligo development is briefly discussed.
\end{abstract}

Keywords: Vitiligo; Melanogenesis; Dysfunction melanocytes; Autoimmune disease

\section{Introduction}

Skin is the largest external organ of the human body and considered as one of the most complicated organ. It has many roles in protecting the human body from surrounding aggressive pathogens and maintains the health and life. Literally, skin can be defined as the layer of commonly soft, flexible outer tissue covering the human or any vertebral animal body, with three main functions: protection, sensation and regulation. The skin consists of three main parts: an outer layer, the epidermis, an inner layer, the corium (or dermis), and the third part is subcutaneous tissues/ hypodermis/subcutis. The outer layer, epidermis, is thinner than the corium, and is made up of several layers of different types of cells. The number of its cells varies in different parts of the body depending on continues contact and fraction with subjects; for example, the greatest number of cells is occurred in the palms of the hands and soles of the feet, where the skin is thicker. The epidermis is composed of keratinized, stratified squamous epithelium. It is made of four or five layers of epithelial cells, based on its location in the body. These layers are classified, from uppermost to innermost, to stratum corneum, stratum granulosum, stratum spinosum and the stratum basale. The stratum basale is a single layer of basal which is very active layer and function as a precursor of the keratinocytes of the epidermis. It also includes two other cell types named merkel and melanocyte cells.

However, corium, located underneath the epidermis, is the thicker part of the skin. It considered as integumentary system which consists of two distinguish connective tissues that interconnected by fibers of collagen extending along. These two components of the dermis are the papillary layer and the reticular layer. The dermal papillae are extending into the upper layer, stratum basale of the epidermis, to form finger-like dermal papillae. Within the papillary layer there are fibroblasts, a small number of fat cells (adipocytes), an abundance of small blood vessels and phagocytes. 
Meanwhile, underlying the papillary layer is the much thicker reticular layer, composed of dense, irregular connective tissue. This layer is supplemented with a dense of blood vessel, sensory and sympathetic nerve. The reticular layer appears reticulated (netlike) due to a tight meshwork of fibers.

The subcutaneous, inner third part of the skin is also named the hypodermal layer or superficial fascia tissues. It is primarily consisting of impenetrable mass of irregular connective tissue that housing large blood vessels and nerve. The subcutaneous layer varies in its thickness all through the body and also from human individual to another. There are a number of structures and specialized cells that exist within subcutaneous tissue, including: collagen and elastin fibers (these attach the dermis to muscles and bones), blood vessels, sebaceous glands, nerve endings, hair follicle roots and fat cells. Fat cell is largely composed the innermost of this layer forming adipose tissue.

\section{Role and Function of Skin}

The principle function of the skin can be summarized into: a. barrier and protective properties, which can be classified into: barrier from physical and mechanical damage, thermal and microbial damages; b. regulate body temperature; c. immunological function; d. metabolic function; and e. sensation and communication.

\section{a. Barrier and protective function}

The function of the skin's barrier is to protect the skin and the entire body from any damage.

i. Physical and mechanical barrier: Protects the body from mechanical damage i.e. friction and impact. Elastin cells of the skin allow skin to recover its shape after deformation. Subcutaneous tissue also proved its ability to absorbs shock. Skin is mainly impermeable to water.

ii. Thermal barrier: Skin is poor conductor of heat; accordingly, it is a good barrier to heat injury and tolerating temperature less than $40^{\circ} \mathrm{C}$. However, higher temperatures may cause marked tissue destruction. Thick epithelial cells cover of palms and soles can be barrier from severe burns. Deep epidermal appendages have also protective function.

iii. Antimicrobial barrier: Skin protects from bacteria, fungi and viruses through special cells named keratinocytes which provide physical barrier and produce cytokines. Meanwhile, commensal bacteria/yeasts, exist on epidermal layer, play a role against microbial pathogens.

iv. Chemical barrier: The skin protects the tissues of the body from chemical damage through keratinocytes that act as chemical barrier. Excessive exposure causes irritant dermatitis and chemical burns.
Therefore, the skin plays a role for body protection from any external exposure that can cause harmful effect.

v. Protective function: As a result for what are mentioned above, the skin performs a protective function against biological (bacteria, viruses, yeast and fungi), physical, and chemical agents. An alkaline substance placed on the skin is neutralized by the hydrolipid film and the horny layer before it can damage the organs below. In the same way the sun's radiation is neutralized, at least in part, by melanin or by the horny layer. Finally, the skin plays the essential role of mechanical protection that we all appreciate every day when large or small mechanical shocks are mitigated by our skin. Meanwhile, it protects the body from losing too much water through evaporation, where, only lipid-soluble substances can penetrate it only if their molecular weight is not disproportionate. This selective permeable barrier is mainly allocated to the basal membrane, horny layer, and intercellular lipids.

\section{b. Regulate body temperature}

Both the skin and the deep body receptors are dealing with body temperature through communication with the anterior and posterior hypothalamus. When body temperature increased sweat glands secrete sweat on the skin, allowing the heat loss through the skin by evaporation. In the meantime, vasodilation of superficial arteries/blood vessels over the entire body, to release internal heat to the air through the skin, will occur. However, when body temperature falls, immediate reflex effects invoked and begin to increase the temperature of the body in several ways: shivering, inhibition of sweating and promoting skin vasoconstriction.

c. Immunological function

The skin is first site of entry for foreign substances and microbes. As mentioned before, the presence of the Langerhan and keratinocyte cells, in skin epidermal layers, and blood and lymph vessels and numerous immune cells, in dermal layers, are able to identify the invaded microbes and able to prepare a defence. The immune system of the skin has elements of both the innate (nonspecific) and adaptive (specific) immune systems that work together in stepwise harmony that lead to protect the body from infection, cancer, toxins, as well as attempts to prevent autoimmunity. The skin microbiome also contributes to the homeostasis of the skin immune system.

\section{d. Metabolic function}

The epidermal layer of the skin is responsible for the synthesis of hormones and vitamins, such as, vitamin The apocrine, eccrine and sebaceous glands are responsible for releasing secretions which metabolically contribute to the barrier function of the skin. 
e. Sensation and communication

Sensation is facilitated by topographically distributed receptors of several types and classes that located in the skin. These receptors are distributed in a grid pattern in the epidermal and dermal layers of the skin. They are also distributed throughout the deep tissues among different regions of skin.

\section{f. Microbiome of the Skin and Risk Diseases}

The term skin microbiome generally is referring to the microorganisms that reside on the human skin. Skin microbiome is covered the body in a non-observed biofilm layer that plays a major role in skin protection. Consistency and diversity of these microorganisms, forming this biofilm, are highly affected by way of two factors: what we put on our skin, and what we put in our body. As we may know that skin health are influenced by the gut microbiome and gut health in general [1,2]. Taking a daily properquality probiotic will help in keeping our skin in health condition. Numerous researches done on the use of probiotics, in supporting a healthy gut and in sequence skin microbiome, proved their significance on human health. New researches also confirmed this relation between gut and skin, and demonstrated that anything damaging our gut microbiome also influences what's happening to the skin [3-4]. This connection is termed the gut-skin axis, and scientists are started to explore such connection to understand this dual effect. The gut and the skin are considered organs with vital immune and neuro-endocrine roles and are uniquely related in persistence and function. The intimate relationship between these organs nowadays, with great concern, has a potential to develop sever diseases like colorectal carcinoma [5,6]. Figure 1 explains the gut-skin possible mechanism for development of different type of skin diseases.

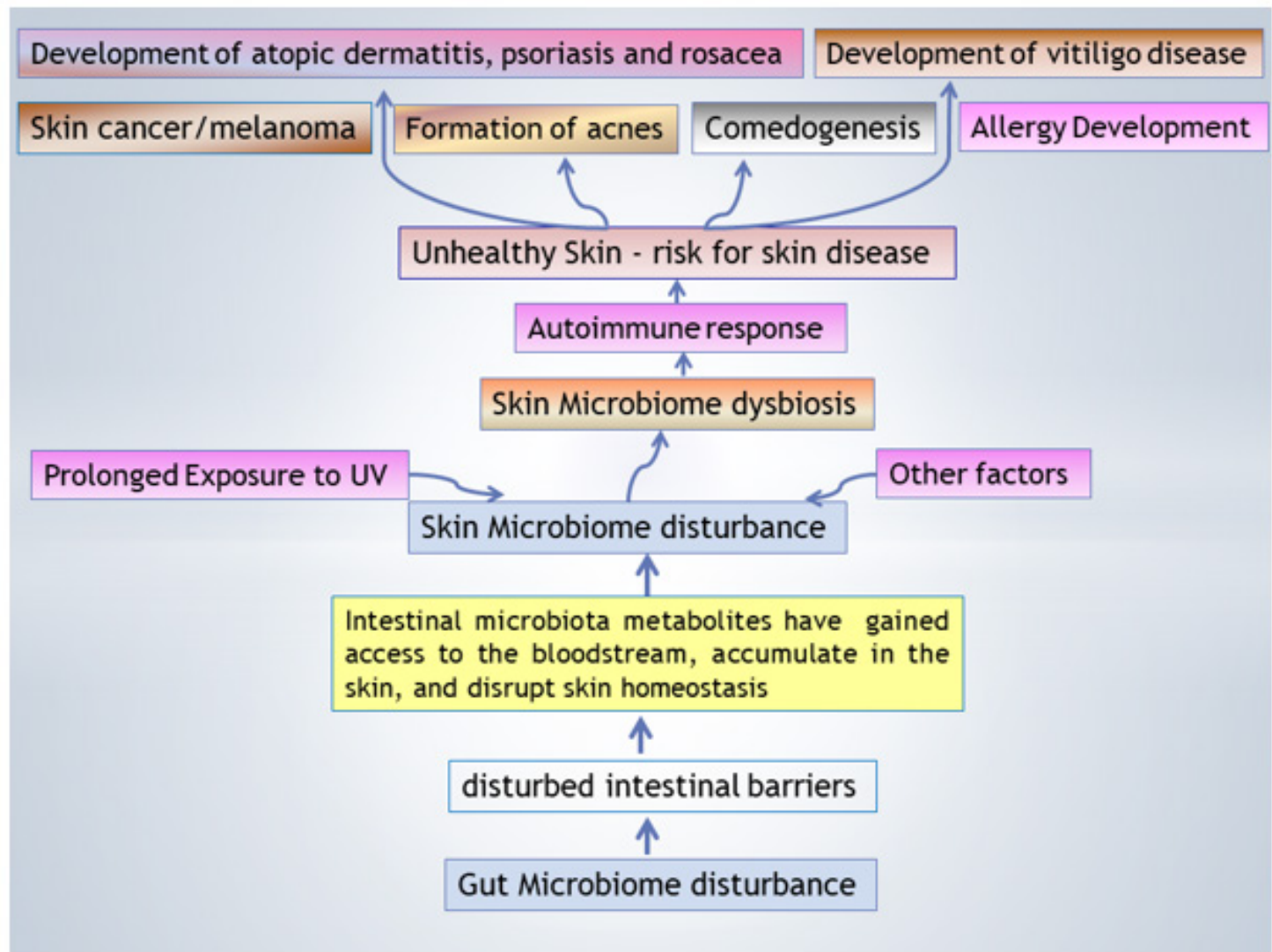

Figure 1: Relation between gut and skin microbiome named "gut-skin axis microbiome" and their influence on skin disease development.

Numerous studies have linked gastrointestinal (GI) health to skin homeostasis. To date, the most research done on acne and its development with reference to gut microbiome disorder [8-10] which proved a strong relation between both of them. However, the lines of communication, as mediated by gut microbes, may be direct and indirect, but ultimately influences the degree of acne severity. Generally, the mechanism by which acne developed through inflammation of the gastrointestinal tract that known as a leaky gut. In this condition, a part of digested food enters the bloodstream, which causes acne breakouts. When the gut microbiome becomes imbalanced or disrupted, everything including the brain to energy level to the skin is affected. Dietary regime also contributed in gut inflammation and dysbiosis of microbiome (Figure 1). To keep the gut microbiome stable state, it is suggested to add prebiotic food 
(such as fiber-rich food, fresh fruits, and vegetables) and probiotic food to the diet to prevent acne development and breakout [11]. There is also early evidence that that potential link between the dysbiosis in the gut microbiome and skin disorders such as psoriasis, rosacea, and atopic dermatitis including dermatitis herpetiformis and Eczema. The gut dysbiosis which lead to leaky gut in which such leakage will cause the immune system to launch an inflammatory response, leading to local and systemic inflammation, which, in turn, contributes to the mentioned disease (Figure 1). In the case of Eczema, the gut microbiota also affect the development of autoimmune disorders, in response the gut microbes regulate $\mathrm{T}$ cell differentiation (and thus, gut microbiome plays an immunoregulatory role) and modify proteins present in the gut, which could produce an autoimmune response $[12,13]$.

\section{Skin Pigmentation and Vitiligo Disease}

Melanocytes, exist in various tissues in the body including the skin, are responsible for melanin pigment production. In the skin, they are localized in the dermal-epidermal interface at the distal epidermal layer. Melanocyte cells have dendrites that projected between epidermal cells where they transfer melanosomes to keratinocytes [14-16]. One melanocyte is associated with nearly 30-40 surrounding keratinocytes through its dendrites. The symbiotic relation between these two cell types, melanocyte and the pool of associated keratinocytes, form an entire unit and is coined as the "epidermal melanin unit". This symbiotic unit plays a role in melanin transfer from melanocyte to keratinocyte cells and gives the skin its color.

For transfer the melanin, from melanocyte to keratinocyte cells, different frequented stages should be occurred by which single melanosomes, the specialized pigment-containing organelles, are released in a keratinocyte cytosol and reach the perinuclear area. Moreover, the amount and type of melanin produced, that transferred to the keratinocytes with subsequent integration, aggregation and degradation, impacts skin complexion coloration
$[17,18]$. Two major classes of melanin are produced pheomelanin, an orange to red pigment, and eumelanin, a brown to black pigment. They differ in their color and the way of synthesis. However, both melanin classes are driven from a single precursor, the amino acid L-tyrosine, and their synthesis involves melanogenic enzymes such as tyrosinase (TYR), a copper-dependent enzyme; tyrosinase-related protein 1 (TYRP1); and tyrosinase-related protein-2 or dopachrome tautomerase (TYRP2) [19,20]. Therefore, the multistep process, which in need to allow the production of melanin pigment of different classes, is called melanogenesis. Regulation of melanogenesis is controlled by transcript factor named microphthalmia-associated transcription factor (MIFT) with others that belong to MiT family factors [20]. These factors regulate the expression of specific genes, from promoters containing specific sequences, for proteins involved in pigment synthesis, for melanosome structural proteins, as well as proteins involved in melanosome trafficking which responsible for melanin production [21-23]. Therefore, any defect of these sequences for melanin production will attribute for skin decolorization.

\section{Vitiligo and Skin Microbiome}

Simply vitiligo is skin disease and can be identified as an autoimmune disease that gives rise to patches of white skin as a resulted from melanocyte destruction. As we mentioned above, gut-skin axis microbiome play a great role in skin diseases which related to disturbance in their normal microbiome. Therefore, gut dysbiosis lead to dysbiosis of skin microbiome and in sequence affect immune system resulted in many different diseases including vitiligo (Figure 2). Many other factors can also affect diversity of skin microbiome and generate skin diseases. Vitiligo can be developed at any age once the melanocyte dysfunction; however, approximately one-half of cases are diagnosed either in childhood or at begging of adult stat before turns 20. Vitiligo can also develop anywhere on human body including on the face, hands, arms, backside and genitals.

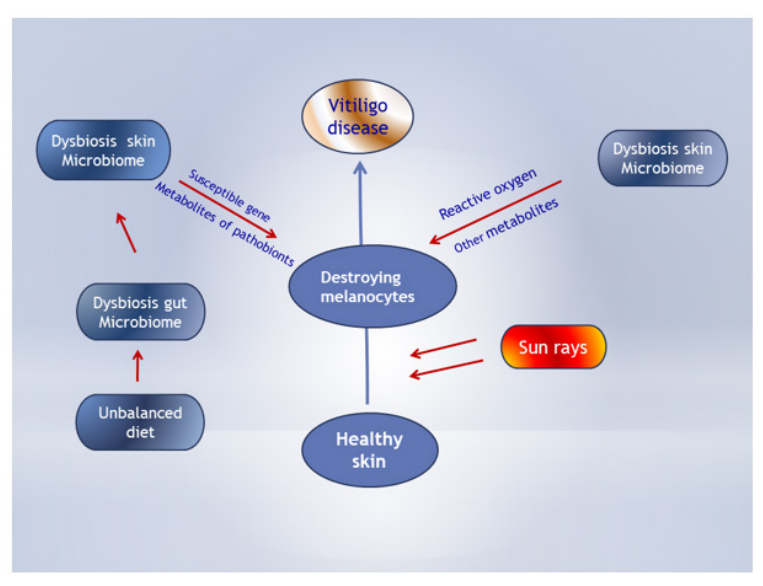

Figure 2: Vitiligo development and suggested theories for the disease development. 
Few studies had been carried out to explore the real causes of melanocyte dysfunction and appearance of white patches on skin [24-26]. Most of the studies done referred to the disturbance of skin microbiome which plays a crucial role in modulating the innate immune that induces activation of suppressor $\mathrm{T}$ cells and lowers cell-mediated immunity. Subsequently, productions of several proinflammatory cytokine mediators by CD4+ and/or CD8+ $\mathrm{T}$ cells were induced which attack the melanocytes and cause its destruction. Some other studies explored the diversity and types of the microbiome of normal skin versus skin lesion of vitiligo patient and showed that alternation in skin microbe leads to domination of some microbes than other. The metabolites of these microbes have an oxidative stress on melanocyte cells or on the melanogenesis process itself and destruct it at any phase (Figure 2) Most importantly, mechanistic studies in mouse models of vitiligo and others, done by Rashighi et al. [27]; Collins et al. [28] and Jillian et al. [29], have specifically implicated an interferon (IFN) $\gamma$-driven immune response, including IFN $\gamma$, IFN $\gamma$-induced chemokines, and cytotoxic CD8 $\mathrm{T}$ cells as the main drivers of disease pathogenesis. Another theory is vitiligo disease results from a complex interaction of environmental, genetic, and immunologic factors, which ultimately contribute to melanocyte destruction, resulting in the characteristic depigmented lesions [30,31]. All of these hypothesis lead to destroying the melanocytes and sequentially the melanin production which ended with appearance of white patches.

\section{Conclusion}

In conclusion, our review may shad a light on the important of skin microbiome, which highly related to gut microbiome and immune system homeostasis, and its role in vitiligo development. Meanwhile, host-microbe interactions and genetically determined the variation of skin properties might be linked to skin dysbiosis. The alterations in skin microbiome also play a role in cellular stress on melanocytes and destruction of melanosome or the melanogenesis process that lead to vitiligo pathophysiology state.

\section{References}

1. Salem I, Ramser A, Isham N, Ghannoum MA (2018) The Gut Microbiome as a Major Regulator of the Gut-Skin Axis. Frontiers in microbiology 9: 1459

2. Heba N Gad EL-Hak, Abel Raouf AM, Samira RM (2018) The Collateral Effect of Antibiotic on Gut Microbiome of Human and their Integrative Role in Human Disease. Adv Res Gastroentero Hepatol 10(3): 555789.

3. Szántó M, Dózsa A, Antal D, Szabó K, Kemény L, et al. (2019) Targeting the gut-skin axis-Probiotics as new tools for skin disorder management? Exp Dermatol 28(11): 1210-1218.

4. Moskovicz V, Gross A, Mizrahi B (2020) Extrinsic Factors Shaping the Skin Microbiome. Microorganisms 8(7): 1023.

5. Casterline BW, Paller AS (2020) Early development of the skin microbiome: therapeutic opportunities. Pediatr Res.

6. Zeller G, Julien T, Anita YV, Shinichi S, Jens RK, et al. (2014) Potential of fecal microbiota for early-stage detection of colorectal cancer. Mol Syst Biol 10(11): 76610.
7. O'Keefe SJD (2016) Diet, microorganisms and their metabolites, and colon cancer. Gastroenterology \& hepatology 13(12): 691-706.

8. Grice EA, Segre JA (2011) The skin microbiome Nat Rev Microbiol 9(4): 244-253.

9. Ganju P, Nagpal S, Mohammed MH, Nishal Kumar P, Pandey R, et al. (2016) Microbial community profiling shows dysbiosis in the lesional skin of Vitiligo subjects. Scientific reports 6: 18761.

10. Santoro A, Zhao J, Wu L, Carru C, Biagi E, et al. (2020) Microbiomes other than the gut: inflammaging and age-related diseases. Seminars in Immunopathology 42(5): 589-605.

11. Darren MB (2011) Probiotics for the Treatment of Adult Gastrointestinal Disorders. Northwestern University, Feinberg School of Medicine, Chicago, USA

12. Campisi L, Barbet G, Ding Y, Esplugues E, Flavell RA, et al. (2016) Apoptosis in response to microbial infection induces autoreactive TH17 cells. Nat Immunol 17(9): 1084-1092.

13. Manfredo SV, Hiltensperger M, Kumar V, Zegarra-Ruiz D, Dehner C, et al. (2018) Translocation of a gut pathobiont drives autoimmunity in mice and humans. Science 359(6380): 1156-1161.

14. Slominski A, Tobin DJ, Shibahara S, Wortsman J (2004) Melanin pigmentation in mammalian skin and its hormonal regulation. Physiol Rev 84(4): 1155-1228.

15. Videira IF, Moura DF, Magina S (2013) Mechanisms regulating melanogenesis. Anais brasileiros de dermatologia 88(1): 76-83.

16. Serre C, Busuttil V, Botto JM (2018) Intrinsic and extrinsic regulation of human skin melanogenesis and pigmentation. Inter J of cosmetic Science $40(4): 328-347$

17. Ebanks JP, Wickett RR, Boissy RE (2009) Mechanisms regulating skin pigmentation: the rise and fall of complexion coloration. International journal of molecular sciences 10(9): 4066-4087.

18. Domingues L, Hurbain I, Gilles-Marsens F, Sirés-Campos J, André N, et al. (2020) Coupling of melanocyte signaling and mechanics by caveolae is required for human skin pigmentation. Nature communications 11(1): 2988.

19. Kondo T, Hearing VJ (2011) Update on the regulation of mammalian melanocyte function and skin pigmentation. Expert Rev Dermatol 6(1): 97-108.

20. Wu Po-Yuan, You Ya-Jhen, Liu Yi-Jung, Hou Chien Wei, Wu Chin-Sheng, et al. (2018). Sesamol Inhibited Melanogenesis by Regulating Melanin-Related Signal Transduction in B16F10 Cells. International Journal of Molecular Sciences 19(4): 1108.

21. Lachmann A, Xu H, Krishnan J, Berger SI, Mazloom AR, et al. (2010) ChEA: transcription factor regulation inferred from integrating genomewide ChIP-X experiments. Bioinformatics 26(19): 2438-2444.

22. Vachtenheim J, Borovanský J (2010) “Transcription physiology” of pigment formation in melanocytes: central role of MITF. Exp Dermatol 19(7): 617-627.

23. Stuckert A, Moore E, Coyle KP, Davison I, MacManes MD, et al. (2019) Variation in pigmentation gene expression is associa ted with distinct aposematic color morphs in the poison frog Dendrobates auratus. BMC evolutionary biology 19(1): 85.

24. Zeeuwen Patrick LJM, Kleerebezem M, Timmerman HarroM, Schalkwijk J (2013) Microbiome and skin diseases. Current Opinion in Allergy and Clinical Immunology 13(5): 514-520.

25. Guerra L, Dellambra E, Brescia S, Raskovic D (2010) Vitiligo: pathogenetic hypotheses and targets for current therapies (2010) Curr Drug Metab 11(5): 451-467.

26. Bondanza S, Riccardo M, Patrizia P, Eleonora M, Fabio DG, et al. (2007) Keratinocyte cultures from involved skin in vitiligo patients show an impaired in vitro behaviour. Pigment Cell Res 20(4): 288-300. 
27. Rashighi M, Agarwal P, Richmond JM, Tajie HH, Karen D, et al. (2014) CXCL10 is critical for the progression and maintenance of depigmentation in a mouse model of vitiligo. Sci Transl Med 6(223): 223ra23.

28. Collins N, Jiang X, Zaid A, Bethany LM, Jane L, et al. (2016). Skin CD4+ memory $\mathrm{T}$ cells exhibit combined cluster-mediated retention and equilibration with the circulation. Nat Commun 7: 11514

29. Jillian M, Richmond James P, Strassner Kingsley I Essien, John E Harris (2019) T-cell positioning by chemokines in autoimmune skin diseases 289(1): 186-204.
30. McCully ML, Kouzeli A, Moser B (2018) Peripheral tissue chemokines: homeostatic control of immune surveillance $\mathrm{T}$ cells. Trends Immunol 39(9): 734-747.

31. Harris JE, Harris TH, Weninger W, Wherry EJ, Hunter CA, et al. (2012) A mouse model of vitiligo with focused epidermal depigmentation requires IFN- $\gamma$ for autoreactive CD8+ T-cell accumulation in the skin. J Invest Dermatol 132(7): 1869-1876. 